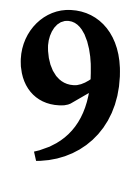

\title{
EFICACIA DE UN PROGRAMA DE INTERVENCIÓN PARA MEJORAR LA COMPETENCIA, AUTONOMÍA Y BIENESTAR DE EDUCADORES DE CENTROS DE PROTECCIÓN DE MENORES
}

\author{
(TESTING AN INTERVENTION TO IMPROVE THE COMPETENCE, \\ AUTONOMY AND WELL BEING OF EDUCATORS IN CENTERS FOR THE \\ PROTECTION OF MINORS)
}

Marisa García-Merita, Isabel Balaguer, Isabel Castillo, Marisol Álvarez y Lidón Mars

Universitat de València

\section{RESUMEN}

En este estudio cuasi-experimental pre-test post-test sin grupo control, se analizaron los efectos de un programa de intervención, cuyo objetivo fue mejorar el bienestar de los educadores a través de la satisfacción de las necesidades de competencia y de autonomía. Participaron 55 educadores, 24 hombres y 31 mujeres $(M$ edad $=33,6 ; D T=8,1)$ que trabajaban en Centros de Protección de Menores de la Comunidad Valenciana. Los resultados mostraron un cambio significativo en todas las variables en la dirección esperada. Además, informaron que los cambios en la satisfacción de las necesidades de competencia y autonomía produjeron cambios en la autoestima; mientras que las variaciones en la vitalidad subjetiva fueron explicadas a partir del incremento en la satisfacción de la necesidad de autonomía. Los resultados sugieren que se puede promover el bienestar de los educadores a través de cambios en la satisfacción de las necesidades básicas.

\section{ABSTRACT}

In this quasi-experimental pre-test post-test study without a control group, the effects of a training program were analyzed. The aim of the intervention was to improve educators' psychological well-being through increasing their autonomy and competence need satisfaction realized in their work with young people. Participants were 55 educators, 24 males and 31 females $(M$ age = 33.6; $S D=8.1$, who worked at different Centers for Protection of Minors from 
Valencian Community. Results reported a significant change, in the expected direction, in all the variables studied. Also, results revealed changes in the satisfaction of the needs for autonomy and competence to be significant predictors of changes in self-esteem. Variations in reported subjective vitality over time were explained by increases of autonomy need satisfaction. The results are aligned with Self Determination Theory and suggest that the welfare of educators involved in protection centers for children can be promoted via increased satisfaction of basic needs.

\section{INTRODUCCIÓN}

En los centros de protección de menores los educadores constituyen la principal figura de referencia tanto para el niño o el adolescente como para la familia y para todas aquellas personas relacionadas con cada joven que reside en el centro (Fernández del Valle y Fuertes, 2000). Su trabajo es de vital importancia ya que ellos centralizan la información de cada caso y asumen la responsabilidad de su educación tomando el rol de "padres sustitutos" (Whittaker y Maluccio, 2002).

Los educadores viven situaciones altamente estresantes que se caracterizan por los bajos salarios, turnos de trabajo con exceso de horas (Schaufeli y Buunk, 2003), la ambigüedad y el conflicto de rol (Landsman, 2001), así como la falta de reconocimiento social y la escasez de recursos (Soderfeldt, Soderfeldt y Warg, 1995), entre otras. Estas condiciones favorecen la incidencia de una elevada tasa de burnout como constatan algunos estudios (p. e., Anderson, 2000). Sin embargo, el burnout, que para algunos autores es considerado como una consecuencia específica del estrés crónico en el trabajo (p. e., Schaufeli y Buunk, 2003), no siempre se da en los profesionales de los centros de protección de menores. Varios estudios han sugerido que existen una serie de variables que modulan este efecto: las características individuales de los educadores, el sentirse recompensados por estar ayudando a otros, asumir el compromiso de tener que velar por el bienestar de los niños y creer que la labor propia es la que "marca la diferencia". Todas estas condiciones cuyo denominador común gira en torno a la lucha por la consecución del bienestar de los menores, contribuyen a que los educadores se sientan satisfechos en su trabajo a pesar de la carga que este supone y del agotamiento emocional que produce (Stalker, Mandell, Frensch, Harvey y Wright, 2007).

Dada esta situación de altas demandas en el puesto de trabajo, varios estudios han sugerido que se elaboren vías de intervención para combatir los efectos de dichas situaciones y para mejorar las condiciones personales y sociales de estos profesionales. Así por ejemplo, Milfont y sus colegas (Milfont, 
Denny, Ameratunga, Robinson y Ferry, 2008) sugieren la conveniencia de realizar intervenciones orientadas a la mejora de su bienestar, mientras que Moses (2000) enfatiza la importancia de promover y mantener la motivación de estos profesionales.

Una de las teorías psicológicas que nos ofrece las vías para desarrollar tanto los recursos psicológicos de los educadores, como para promover su motivación y para potenciar su bienestar es la teoría de la autodeterminación (SDT; p. e., Deci y Ryan, 2000; Ryan y Deci, 2000). Esta teoría propone la existencia de tres necesidades psicológicas básicas que se consideran "nutrientes esenciales para el crecimiento psicológico, la integridad y el bienestar" (Deci y Ryan, 2000, p. 229): La necesidad de competencia (se refiere al deseo de actuar de forma eficaz con el ambiente), la necesidad de autonomía (se refiere al deseo de implicarse en actividades de propia elección y al deseo de ser uno mismo el iniciador de sus propias conductas), y la necesidad de relación (referida al sentimiento de sentirse conectado con los otros o al sentimiento de pertenecer a un medio social dado) (Deci y Ryan, 1985).

El concepto de necesidad en la SDT se refiere a un estado de energía que si se satisface conduce a la salud y al bienestar pero que en caso contrario contribuye a la patología y al malestar. De ahí que Ryan y Deci (2000) propongan que cuando estas necesidades están satisfechas los sujetos se perciben con bienestar óptimo. Esta hipótesis ha recibido apoyo empírico desde diferentes contextos (p. e., trabajo, deporte, y estudiantes universitarios). Así por ejemplo, en el contexto deportivo, Reinboth, Duda y Ntoumanis (2004) encontraron que la satisfacción de la competencia y de la autonomía actuó de predictor positivo de la vitalidad subjetiva. En el contexto del trabajo, la satisfacción de las necesidades de competencia, de autonomía y de relación, estaba asociada con más vitalidad y menos ansiedad y síntomas físicos (Baard, Deci y Ryan, 2000). En el contexto educativo se encontró que la satisfacción de las necesidades de autonomía, competencia y relación estaba positivamente asociada con la autoestima, con los afectos positivos y con la vitalidad, y negativamente asociado con los afectos negativos (Patrick, Knee, Canevello y Lonsbary, 2007). Recientemente, en el contexto de los centros de protección de menores, García-Merita y sus colegas (García-Merita, Balaguer, Castillo, Álvarez y Mars, 2009) realizaron un estudio transversal con educadores en el que encontraron que la satisfacción de las necesidades de competencia, autonomía y relación estaba positivamente relacionada con la vitalidad subjetiva, la autoestima y la satisfacción con la vida.

El presente trabajo tuvo como objetivo analizar el impacto de un programa de formación, destinado a mejorar el bienestar de los educadores a 
través de la satisfacción de las necesidades de competencia y de autonomía. No se introdujo la necesidad de relación (en el centro de trabajo), debido a que la intervención se realizaba con otros compañeros de otros centros y por consiguiente no existía la opción de apreciar el cambio en esta variable. Siguiendo investigaciones previas los indicadores del bienestar fueron la vitalidad subjetiva y la autoestima (p.e., García-Merita et al., 2009; Patrick et al., 2007; Reinboth et al., 2004; Reinboth y Duda, 2006).

Siguiendo a Deci y Ryan (2000) se hipotetizó que la aplicación de un programa de intervención, produciría sus efectos (diferencias antes-después) sobre la satisfacción de las necesidades de autonomía y de competencia y sobre los indicadores de bienestar. Además se hipotetizó que las variaciones en la autonomía y la competencia tras la administración del programa predecirían directamente diferencias en el bienestar psicológico de los educadores.

Dada la importancia que tiene el bienestar de los educadores de los centros de protección de menores, tanto para su satisfacción personal como para un adecuado desarrollo de su función educativa con menores especialmente sensibles, consideramos que es fundamental llegar a entender los procesos antecedentes que actúan de predictores del bienestar, así como conocer si esas variables antecedentes pueden modificarse mediante una intervención. También queremos examinar si una vez modificadas dichas variables se producen cambios en los indicadores del bienestar psicológico.

\section{MÉTODO}

\subsection{Participantes}

Los participantes en el presente estudio fueron 55 educadores (24 hombres y 31 mujeres) con edades comprendidas entre los 23 y los 58 años $(M=33.55 ; D T=8.1)$, pertenecientes a diferentes Centros de Protección de Menores de la Comunidad Valenciana.

La muestra se obtuvo de un grupo de educadores que realizaban de forma voluntaria un programa de formación organizado por la Consellería de Bienestar Social en colaboración con la Universidad de Valencia. Concretamente se realizó un curso en Valencia, uno en Alicante y uno en Castellón. Todos con el mismo contenido y metodología, e impartidos por la misma persona. Los educadores, que participaron voluntariamente en este estudio, pertenecían a distintas tipologías de centros de protección de menores de las provincias de Valencia, Castellón y Alicante. 


\subsection{Instrumentos}

La satisfacción de la necesidad de competencia se midió con la versión castellana (Balaguer, Castillo y Duda, 2008) de la Escala de Competencia Percibida del Cuestionario de Motivación Intrínseca (McAuley, Duncan, y Tammen, 1989), adaptada para los educadores de centros de protección. Esta escala compuesta por 6 ítems evalúa la percepción de competencia como educador en el centro. Se solicita a los educadores que indiquen el nivel de acuerdo con afirmaciones como "Creo que soy bastante bueno/a educando a los jóvenes de este centro". Los educadores contestan en una escala tipo Likert de 7 puntos, que oscila desde nada verdadero (1) hasta muy verdadero (7).

La satisfacción de la necesidad de autonomía se evaluó con la versión castellana (Balaguer et al., 2008) de la Escala de Autonomía Percibida (Reinboth y Duda, 2006) adaptada para los educadores de centros de protección. Esta escala compuesta por 10 ítems evalúa dos facetas de la autonomía percibida por el educador en el centro: la elección / toma de decisión y los aspectos volitivos. Se solicita a los educadores que indiquen, en general, cómo se sienten cuando educan a los jóvenes en el centro, por ejemplo, "Siento que mi opinión se tiene en cuenta a la hora de decidir cómo se debe llevar a cabo la educación" (elección/toma de decisión), y "Me siento libre de hacer las cosas a mi manera" (aspecto volitivo). Los educadores contestan en una escala tipo Likert de 7 puntos, con un rango que oscila desde nada verdadero (1) hasta muy verdadero (7).

La vitalidad subjetiva se evaluó con la versión castellana (Balaguer, Castillo, García-Merita, y Mars, 2005) de la Escala de Vitalidad Subjetiva (Ryan y Frederick, 1997), que mide los sentimientos subjetivos de energía y viveza. La escala consta de 6 ítems en los que se pide que se indique el grado en que, por lo general, una serie de afirmaciones son verdaderas. Contiene ítems del tipo "Me siento vivo y vital". Las respuestas se recogen en una escala tipo Likert de 7 puntos que oscila desde no es verdad (1) a verdadero (7).

La autoestima se evaluó con la versión castellana (Atienza, Moreno y Balaguer, 2000) de la Escala de Autoestima (Rosenberg, 1965), que evalúa la autoestima global a través de 10 ítems que reflejan sentimientos generales sobre el yo (p. e., "Me siento una persona tan valiosa como las otras"). Las respuestas se recogen en una escala tipo Likert de 4 puntos que oscila desde muy en desacuerdo (1) a muy de acuerdo (4). 


\subsection{Diseño y Procedimiento}

El diseño cuasi-experimental que se ha utilizado en el estudio es el de pretest-postest con un solo grupo. El grupo de educadores cumplimentó los cuestionarios para medir las variables: percepción de competencia, percepción de autonomía, vitalidad subjetiva y autoestima, antes de la intervención (pretest - Tiempo 1-) y una vez que esta finalizó (postest — Tiempo 2-).

El programa de intervención consistió en un curso de formación de veintiocho horas repartidas en seis sesiones de aproximadamente cuatro horas y media cada una, realizadas a la largo de dos semanas.

\subsection{Diseño y contenidos del Programa}

El programa de formación que se aplicó en este trabajo, consistió en ofrecer a los educadores vías a través de las cuales pudiesen generar estrategias educativas que les permitiesen afrontar mejor sus funciones en el entorno laboral. Concretamente se les instruyó en la creación de climas motivacionales positivos.

Dicho programa que contenía aspectos teórico-prácticos basados en la SDT (p.e., Ryan y Deci, 2000) y en la Teoría de las Metas de Logro (p.e., Ames, 1992), se realizó en la modalidad de taller, con la finalidad de que los educadores tuvieran una participación activa y pudieran aprovechar las ventajas del trabajo en equipo.

Se llevaron a cabo tres talleres, dos talleres para adquirir las habilidades necesarias para crear climas de apoyo a la autonomía, y un taller para aprender a promover climas de implicación en la tarea.

\section{A) Clima de apoyo a la autonomía}

El apoyo a la autonomía significa desarrollar en los niños un estilo de enseñanza que consiga potenciar la motivación intrínseca y los procesos de internalización. Eso conlleva creencias específicas sobre la forma de motivarlos, una orientación interpersonal particular en el proceso de formación y la posesión de una serie de habilidades interpersonales (Reeve, 2002).

Respecto a las creencias especificas sobre la forma de motivar a los niños, a los educadores se les enseñó a diferenciar entre los estilos controladores y los estilos de apoyo a la autonomía, enfatizando la importancia de estos últimos. 
La orientación interpersonal particular de apoyo a la autonomía se caracteriza por la siguiente predisposición (a) ponerse en la perspectiva de los niños para apoyar su iniciativa, (b) desarrollar su competencia, y (c) relacionarse con un lenguaje que fuese auténticamente informativo y no controlador o impositivo.

El primer taller se realizó con el objetivo de que los educadores adquiriesen tanto las creencias sobre la forma de motivar a los niños, desde la promoción de un clima de apoyo a la autonomía, como las habilidades para desarrollar en los centros una orientación interpersonal hacia la creación de este tipo de atmósfera. En este taller se entrenó a los educadores en las siguientes habilidades interpersonales: (1) ponerse en la perspectiva de los otros, (2) reconocer las emociones, (3) ofrecer argumentos que le diesen valor a aquellas cosas o tareas que no les resultaban interesantes y (4) estar abiertos a la expresión de afectos negativos.

La metodología de trabajo de este taller consistió en presentar, en primer lugar, los contenidos básicos de la SDT (p. e., tipos de motivación, estilos de interacción educador-niño). En segundo lugar, se presentaron los apoyos empíricos de esta teoría en el contexto educativo, destacando los beneficios que podría generar este tipo de ambiente social sobre el bienestar y los estilos de vida de los jóvenes en situaciones de protección social (Ryan y Deci, 2000). En el tercer paso, se proporcionaron ilustraciones a través del visionado de escenas de películas, para que identificasen cada una de las cuatro estrategias de apoyo a la autonomía mencionadas. En el cuarto paso, los educadores trabajaron en pequeños grupos y llevaron a cabo pautas concretas para mejorar cada una de esas cuatro estrategias de apoyo a la autonomía. Finalmente, este primer taller concluyó con una discusión de todo el grupo acerca de la viabilidad, aplicación y obstáculos de cada una de esas cuatro estrategias de apoyo a la autonomía en su propia realidad laboral.

El segundo taller tuvo como objetivo profundizar en los aspectos centrales de la creación de un ambiente social de apoyo a la autonomía, centrándonos específicamente en el desarrollo de las siguientes habilidades interpersonales: (a) gestionar las emociones de los diferentes grupos de niños/jóvenes con los que trabajaban; en concreto se trabajaron las capacidades de la autoconciencia emocional y de la regulación de las emociones, (b) entrenamiento en el desarrollo de estrategias de comunicación con el grupo para fomentar las habilidades de la escucha activa y la comunicación asertiva, y (c) desarrollo de la empatía, trabajando la capacidad para comprender y anticiparse a las necesidades y sentimientos de los demás respondiendo adecuadamente a sus reacciones emocionales. 
La metodología de este segundo taller consistió, en primer lugar, en profundizar en aquellos aspectos de la SDT referidos a las relaciones interpersonales de apoyo a la autonomía, descritas anteriormente. En segundo lugar, se realizaron una serie de ejercicios individuales para estimular la reflexión personal. También se utilizó el visionado de escenas de películas para tomar conciencia de qué actitudes, estrategias y enfoques podían resultar útiles y cuáles inútiles en la labor del educador. Otra estrategia de aprendizaje utilizada para mejorar las destrezas y habilidades emocionales, de comunicación y de empatía fue la realización de role-playing.

\section{B) Clima de Implicación en la Tarea}

En el marco de la teoría de las metas de logro (Ames, 1992) se considera que el clima motivacional percibido creado por los educadores se caracteriza por un conjunto de facetas que incluyen tanto la estructura situacional como los estándares de los criterios que utilizan los educadores en su dinámica con los niños durante el proceso de formación en distintas actividades. Ames (1992) propuso que el clima motivacional es multidimensional y está compuesto por diferentes estructuras, que se recogen en el acrónimo TARGET (diseño de la Tarea, fuente de Autoridad, naturaleza del Reconocimiento, forma de aGrupar, cómo se realiza la Evaluación y el Tiempo ofrecido para ejecutar las tareas) y que reflejan las características de la estructura del ambiente que define el clima que crean las fuentes de autoridad. Sus investigaciones en el ámbito de la educación mostraron que los educadores creaban al menos dos tipos de climas, uno de implicación en la maestría o en la tarea y uno de implicación en el resultado o en el ego. Comprobaron que el primero de ellos era el que potenciaba la adquisición de las estrategias más adaptativas en los estudiantes (Ames, 1992). La investigación posterior en el contexto educativo ha continuado ofreciendo apoyo empírico sobre los beneficios del clima de implicación en la tarea para el buen funcionamiento de los estudiantes (Meece, Anderman, y Anderman, 2006).

Para que los educadores aprendiesen a diseñar climas motivacionales de implicación en la tarea se realizó un tercer taller en el que se les enseñó a utilizar las estrategias del TARGET adaptadas por Ames (1992) al contexto de la educación (véase Balaguer, 2007).

La metodología de este taller consistió, en primer lugar, en instruir a los educadores sobre los contenidos básicos de la teoría de las metas de logro así como sobre los aspectos teórico-aplicados de las estructuras TARGET. A continuación se les informó sobre los apoyos empíricos de esta teoría en el contexto educativo para mejorar el desarrollo positivo de los estudiantes, tanto a nivel de rendimiento, como a nivel emocional y cognitivo (Ames, 1992). 
Los siguientes pasos, al igual que en los talleres anteriores, consistieron en el visionado de escenas de películas para que identificasen los tipos de clima motivacional, el trabajo en pequeños grupos para diseñar una estructura TARGET de implicación en la tarea especifica de sus lugares de trabajo, y la discusión de todo el grupo sobre la viabilidad, aplicación y obstáculos del desarrollo del clima de implicación en la tarea en su propia realidad laboral.

\section{RESULTADOS}

En la Tabla 1 se muestran las medias, las desviaciones típicas y los coeficientes alfa de todas las variables del estudio, tanto para el Tiempo 1 como para el Tiempo 2. Todos los coeficientes de fiabilidad se encuentran por encima del criterio de 0,70 , oscilando el rango entre 0,71 y 0,92 .

Con la finalidad de evaluar los efectos del programa de intervención sobre las variables estudiadas se realizó una prueba $t$ para muestras dependientes. Los resultados mostraron diferencias estadísticamente significativas en todas las variables evaluadas, produciéndose un aumento significativo en todas las puntuaciones entre el Tiempo 1 y el Tiempo 2 en la dirección esperada (véase Tabla 1). Los mayores cambios se produjeron en la satisfacción de las dos necesidades estudiadas, siendo la mayor de ellas la satisfacción de la necesidad de competencia.

\begin{tabular}{|l|c|c|c|c|c|c|c|c|}
\hline \multirow{2}{*}{ Variables } & \multicolumn{4}{|c|}{ Tiempo 1 } & \multicolumn{4}{c|}{ Tiempo 2 } \\
\cline { 2 - 9 } & Rango & $\boldsymbol{M}$ & $\boldsymbol{D T}$ & $\boldsymbol{\alpha}$ & $\boldsymbol{M}$ & $\boldsymbol{D T}$ & $\boldsymbol{\alpha}$ & $\boldsymbol{t}$ \\
\hline Necesidad Competencia & $1-7$ & 4,32 & 0,76 & 0,85 & 5,53 & 0,73 & 0,87 & $-11,90 * *$ \\
\hline Necesidad Autonomía & $1-7$ & 4,77 & 0,79 & 0,92 & 5,23 & 0,85 & 0,90 & $-7,17 * *$ \\
\hline Vitalidad Subjetiva & $1-7$ & 4,99 & 0,97 & 0,87 & 5,31 & 0,90 & 0,88 & $-3,36 * *$ \\
\hline Autoestima & $1-4$ & 3,39 & 0,34 & 0,71 & 3,54 & 0,35 & 0,79 & $-2,77 * *$ \\
\hline
\end{tabular}

$* * p<0,01 ; * p<0,05$

Tabla 1. Descriptivos, Consistencia interna y Diferenciales de cada medida

Se realizaron análisis de regresión jerárquica para comprobar si la satisfacción de las necesidades de competencia y de autonomía nos permitía predecir cambios en el bienestar psicológico, tras la intervención. Se utilizó el siguiente procedimiento: En el Paso 1 se introdujeron la puntuación obtenida en el Tiempo 1 en la variable de bienestar a predecir (vitalidad subjetiva o autoestima) y la puntuación de la satisfacción de las necesidades de competencia y de autonomía del Tiempo 1. En el Paso 2 se introdujeron las puntuaciones de la satisfacción de las necesidades de competencia y de au- 
tonomía percibidas del Tiempo 2. Este acercamiento jerárquico ofrece información sobre la forma en la que los cambios producidos en la satisfacción de las necesidades de competencia y de autonomía se asocian a cambios en el bienestar psicológico de los educadores, controlando estadísticamente las puntuaciones obtenidas en el Tiempo 1. Esto es, las puntuaciones en las variables necesidad de competencia y necesidad de autonomía en Tiempo 2, pueden explicar la varianza residual de cada variable de interés (vitalidad subjetiva o autoestima) que no ha sido explicada por las variables introducidas en el primer paso del análisis jerárquico.

El modelo de regresión jerárquica de la variable vitalidad subjetiva ha resultado significativo tanto en el Paso $1[F(3,51)=18,67, p<0,001]$, como en el Paso $2[F(5,49)=14,86, p<0,001]$. Asimismo, el modelo de regresión jerárquica de la variable autoestima ha resultado significativo tanto en el Paso $1[F(3,51)=5,05, p<0,01]$, como en el Paso $2[F(5,49)=6,67$, $p<0,001]$.

Los resultados mostraron (véase Tabla 2) que un aumento de la satisfacción de la necesidad de autonomía explicó una varianza significativa del aumento de la vitalidad subjetiva $(\beta=0,47, p<0,01)$ y de la autoestima $(\beta=0,56, p<0,05)$. Asimismo, un aumento de la satisfacción de la necesidad de competencia predijo un aumento significativo de la autoestima $(\beta=0,27$, $p<0,05)$. Sin embargo, el aumento de la satisfacción de la necesidad de competencia no se mostró como un predictor significativo del aumento de la vitalidad subjetiva.

\section{DISCUSIÓN}

En el presente trabajo se realizó un estudio cuasi-experimental pre-test pos-test cuyo objetivo principal consistió en investigar si tras la administración de un programa de intervención, diseñado a la base de la teoría de la autodeterminación (Ryan y Deci, 2000) y de la teoría de las metas de logro (p.e., Ames, 1992), se producían cambios en la satisfacción de las necesidades (competencia y de autonomía) y en los indicadores del bienestar psicológico (vitalidad subjetiva y autoestima) de los educadores de centros de protección de menores. No existen trabajos anteriores que hayan analizado esta relación, en el contexto de los centros de protección de menores, y más concretamente con educadores. Esto supone una aportación original de la presente investigación.

Los resultados mostraron que la aplicación del programa de intervención produjo una mejora significativa (diferencias antes-después) en la satisfacción de las necesidades de autonomía y de competencia y en los indi- 


\begin{tabular}{|c|c|c|c|c|c|}
\hline Variables & $\boldsymbol{R}^{2}$ & $\boldsymbol{B}$ & SE $B$ & $\beta$ & $t$ \\
\hline \multicolumn{6}{|c|}{ Vitalidad Subjetiva T2 } \\
\hline Paso 1 & $0,52 * *$ & & & & \\
\hline Vitalidad Subjetiva (T1) & & 0,65 & 0,10 & 0,70 & $6,47 * *$ \\
\hline Autonomía (T1) & & 0,02 & 0,13 & 0,02 & 0,15 \\
\hline Competencia (T1) & & 0,07 & 0,13 & 0,06 & 0,53 \\
\hline Paso 2 & $0,08 * *$ & & & & \\
\hline Vitalidad Subjetiva (T1) & & 0,64 & 0,09 & 0,68 & $6,81 * *$ \\
\hline Autonomía (T1) & & 0,40 & 0,20 & 0,35 & $2,05 *$ \\
\hline Competencia (T1) & & 0,08 & 0,13 & 0,07 & 0,61 \\
\hline Autonomía (T2) & & 0,50 & 0,21 & $0, .47$ & $2,43 * *$ \\
\hline Competencia (T2) & & 0,10 & 0,15 & 0,08 & 0,69 \\
\hline \multicolumn{6}{|c|}{ Autoestima T2 } \\
\hline Paso 1 & $0,23 * *$ & & & & \\
\hline Autoestima (T1) & & 0,34 & 0,13 & 0,33 & $2,60 * *$ \\
\hline Autonomía (T1) & & 0,07 & 0,06 & 0,15 & 1,05 \\
\hline Competencia (T1) & & 0,08 & 0,06 & 0,18 & 1,34 \\
\hline Paso 2 & $0,18 * *$ & & & & \\
\hline Autoestima (T1) & & 0,18 & 0,13 & 0,18 & 1,46 \\
\hline Autonomía (T1) & & 0,13 & 0,09 & 0,29 & 1,39 \\
\hline Competencia (T1) & & 0,02 & 0,06 & 0,03 & 0,24 \\
\hline Autonomía (T2) & & 0,23 & 0,10 & 0,56 & $2,34 *$ \\
\hline Competencia (T2) & & 0,13 & 0,07 & 0,27 & $1,82 *$ \\
\hline
\end{tabular}

$* p<0,05, * * p<0,01$

Tabla 2. Análisis de Regresión Jerárquica para predecir el bienestar psicológico en el Tiempo 2

cadores de bienestar (vitalidad subjetiva y autoestima) evaluados, lo cual refuerza la importancia de esta intervención en la consecución del aumento del bienestar de los educadores.

El cambio producido en la satisfacción de la necesidad de autonomía actuó como predictor del incremento producido en la vitalidad subjetiva tras el programa de intervención, al igual que sucedió en investigaciones previas en otros contextos (p.e., Reinboth y Duda, 2006). Este resultado es acorde a 
las asunciones de Ryan y Frederick (1997), en las que defienden que el locus de causalidad percibido interno puede jugar un papel muy importante en los sentimientos de vitalidad. De forma que un educador que siente que planifica autónomamente los proyectos y actividades a realizar con los niños del centro se percibirá con mayor energía disponible que un educador que lo hace porque lo tiene que hacer, esto es, porque son las fuerzas externas las que dictan su conducta. Sin embargo, contrario a lo hipotetizado, pero en línea con investigaciones previas en otros contextos (p.e., Reinboth y Duda, 2006), las percepciones de competencia no emergieron como predictores de la vitalidad subjetiva tras la intervención. Lo que lleva a interpretar, que lo que realmente incrementa la vitalidad percibida son los sentimientos de autonomía. Esto es, para los educadores lo que realmente aumenta su vitalidad, es decir, sentirse el motor de sus propias acciones, a pesar del duro trabajo, es saber que están allí porque ellos quieren, por sus propios objetivos, y que su trabajo con los niños depende de ellos, no de lo que otros decidan por ellos.

Por su parte, el cambio en la satisfacción de las necesidades de autonomía y de competencia predijo positivamente, tras la intervención, el incremento en la autoestima. Todo ello lleva a considerar que la formación que recibieron los educadores contribuyó al aumento de su autoestima porque perciben que se sienten más capaces de ayudar a los niños de los centros y se valoran más a ellos mismos. Los educadores conocen su trabajo y saben lo que los niños necesitan, pero también saben que su propia labor "marca la diferencia" y que con las herramientas que han recibido en el curso se sienten más competentes para llevarlo a cabo.

Así pues, un resultado relevante de esta investigación y que apunta hacia la aplicabilidad del programa de formación utilizado, es la constatación de que es posible promover el bienestar de los educadores a través de la satisfacción de las necesidades de competencia y de autonomía. Estudios futuros tendrán que poner a prueba si en las intervenciones de este tipo realizadas en los propios centros de protección de menores, el incremento de la satisfacción de la necesidad de relación también actúa como predictor significativo de los cambios del bienestar psicológico.

Los resultados del presente estudio apoyan las proposiciones de Milfont y sus colaboradores (2008) sobre la importancia de realizar intervenciones para mejorar el bienestar de los educadores, y las indicaciones de Moses (2000) sobre la importancia de la intervención en los centros de protección de menores para promover y mantener la motivación de los educadores, ya que por investigaciones previas sabemos que la satisfacción de las necesidades de competencia y de autonomía influyen en la motivación intrínseca de los educadores (Ryan y Deci, 2000). 
Los resultados obtenidos en cuanto a su posible aplicabilidad, dan respuesta a algunas necesidades sociales importantes. En concreto, a la forma en que se pueden favorecer las necesidades psicológicas básicas de los educadores de los centros de protección de menores y su bienestar. Además, la mínima infraestructura y el poco tiempo que se necesita para aplicarlo hacen que consideremos muy recomendable este tipo de programas en un sector tan necesitado de apoyo como es el de los profesionales de los centros de protección de menores. Se sabe que la gran mayoría de los educadores de los centros de protección de menores sufre una gran carga emocional, un sobreesfuerzo y que, en muchas ocasiones, el riesgo de burnout es alto (Moses, 2000, Stalker et al., 2007). Es por ello que el conseguir aumentar el bienestar de los trabajadores de los centros de protección es una de las grandes tareas a la que deben enfrentarse los responsables políticos de esta área de los Servicios Sociales. Como señala García-Merita (1997), no basta que los profesionales del sector estén cualificados, que haya inversión en las infraestructuras de los centros y un proyecto educativo individualizado, es totalmente necesario que dichos profesionales tengan un alto nivel de bienestar.

No se quiere finalizar el trabajo sin hacer mención a que el diseño utilizado no permite poner a prueba las relaciones de causalidad que se apuntan con los análisis estadísticos realizados, pero constituyen un indicio empírico a tener en cuenta en el contexto de la teoría que se está manejando y de los objetivos de la investigación. Sin embargo, se considera que hay que valorar la utilidad que este tipo de investigaciones tienen en el contexto de las "situaciones reales" que son las que se producen en el día a día en el mundo profesional. Pues esto es lo que en definitiva nos permite seguir diseñando intervenciones de campo para las necesidades cotidianas.

Consideramos que la aportación más relevante de esta investigación, ha consistido en comprobar a nivel científico, que mediante programas de intervención relativamente fáciles de aplicar, puede actuarse para favorecer el bienestar de los educadores de centros de protección de menores, dando las claves que nos permitan eliminar el burnout y suplir las necesidades que padecen estos profesionales tales como la escasez de recursos y la falta de reconocimiento social. Al actuar sobre estos profesionales mejorando sus condiciones personales y sociales, que duda cabe que estaremos actuando también sobre los menores que tienen a su cargo, que en último término son el objetivo primordial de este tipo de intervenciones.

\section{NOTA}

Esta investigación ha sido apoyada por la Consellería de Bienestar Social. Generalitat Valenciana (2005-2006). 


\section{REFERENCIAS BIBLIOGRÁFICAS}

Ames, C. (1992). Classrooms, goals, structures, and student motivation. Journal of Educational Psychology, 84, 261-271.

Anderson, D. G. (2000). Coping strategies and burnout among veteran child protection workers. Child Abuse and Neglect, 24, 839-848.

Atienza, F. L., Moreno, Y. y Balaguer, I. (2000). Análisis de la dimensionalidad de la Escala de Autoestima de Rosenberg en una muestra de adolescentes valencianos. Revista Psicología Universitas Tarraconensis, 22, 29-42.

Baard, P. P., Deci, E. L. y Ryan, R. M. (2000). Intrinsic need satisfaction as a motivational basis of performance and well-being at work: An application of cognitive evaluation theory. Unpublished manuscript, Fordham University, New York.

Balaguer, I. (2007). Clima motivacional, calidad de la implicación y bienestar psicológico: una propuesta de intervención en equipos deportivos, en Blanco, A y Rodríguez Marín, J. (coords.), Intervención Psicosocial. Madrid: Pearson Educación, 135-162.

Balaguer, I., Castillo, I. y Duda, J. L. (2008). Apoyo a la autonomía, satisfacción de las necesidades, motivación y bienestar en deportistas de competición: un análisis de la teoría de la autodeterminación. Revista de Psicología del Deporte, 17, 123-139.

Balaguer, I., Castillo, I., García-Merita, M. y Mars, L. (2005). Implications of structured extracurricular activities on adolescent's well being and risk behaviors: motivational mechanisms. $9^{\text {th }} \mathrm{Eu}-$ ropean Congress of Psychology, Granada.
Deci, E. L. y Ryan, R. M. (1985). Intrinsic motivation and self-determination in human behavior. New York: Plenum Press.

Deci, E. L. y Ryan, R. M. (2000). The "what" and "why" of goal pursuits: Human needs and the self-determination of behavior. Psychological Inquiry, 11, 227-268.

Fernández del Valle, J. y Fuertes, J. (2000). El acogimiento residencial en la protección a la infancia. Madrid: Pirámide.

García-Merita, M. (1997). Comparecencia como Directora General de Servicios Sociales en la Comisión de Política Social y Empleo. Diario de sesiones del 4 de febrero pp. 3-17. Cortes Valencianas.

García-Merita, M., Balaguer, I., Castillo, I., Álvarez, M. y Mars, L. (2009). Implicaciones de las necesidades psicológicas en el bienestar de los educadores de centros de protección de menores. Manuscrito en revisión.

Landsman, M.J. (2001). Commitment in public child welfare. Social Service Review, 75, 386-419.

McAuley, E., Duncan, T. y Tammen, V. V. (1989). Psychometric properties of the intrinsic motivation inventory in a competitive sport setting: A confirmatory factor analysis. Research Quarterly for Exercise and Sport, 60, 48-58.

Meece, J., Anderman E. M. y Anderman, L. H. (2006). Structures and goals of educational settings: Classroom goal structure, student motivation, and academic achievement, in Fiske, S. T.; Kazdin, A. E. y Schacter, D. L. (eds.) Annual Review of Psychology, Vol. 57, Stanford, CA: Annual Reviews, 487-504. 
Milfont, T. L., Denny, S., Ameratunga, Sh., Robinson, E. y Merry, S. (2008). Burnout and well-being: Testing the Copenhagen burnout inventory in New Zealand teachers. Social Indicators Research, 89, 169-177.

Moses, T. (2000). Why people choose to be residential child care workers. Child \& Youth Care Forum, 29,113-126.

Patrick, H., Knee, C. R., Canevello, A. y Lonsbary, C. (2007). The role of need fulfillment in relationship functioning and well-being: A self-determination theory perspective. Journal of Personality and Social Psychology, 92, 434-457.

Reeve, J. (2002). Self-determination theory applied to educational settings, en Deci, E. L. y Ryan, R. M. (eds.) Handbook of self-determination research. Rochester, NY: University of Rochester Press, 183-203.

Reinboth, M. y Duda, J. L. (2006). Perceived motivational climate, need satisfaction and indices of well-being in team sports: A longitudinal perspective. Psychology of Sport and Exercise, 7, 269-286.

Reinboth, M., Duda, J. L. y Ntoumanis, N. (2004). Dimensions of coaching behavior, need satisfaction, and the psychological and physical welfare of young athletes. Motivation and Emotion, 28, 3, 297-313.

Rosenberg, M. (1965). Society and the adolescents self-image. Princeton, NJ: Princeton University Press.
Ryan, R. M y Deci, E. L. (2000). Self-determination theory and the facilitation of intrinsic motivation, social development, and well being. American Psychologist, 55, 68-78.

Ryan, R. M. y Frederick, C. M. (1997). On energy, personality, and health: subjective vitality as a dynamic reflection of well-being. Journal of Personality, 65, 529-565.

Schaufeli, W. B. y Buunk, B. P. (2003). Burnout: An overview of 25 years of research and theorizing, en Schabracq, M. J.; Winnubs, J. A. M. y Cooper, C. L. (eds.) The handbook of work and health psychology. New York: Wiley, 383-425.

Soderfeldt, M., Soderfeldt, B. y Warg, L. (1995). Burnout in social work. $\mathrm{Na}$ tional Association of Social Workers Review, 40, 638-646.

Stalker, C. A., Mandell, D., Frensch, K. M., Harvey, Ch. y Wright, M. (2007). Child welfare workers who are exhausted yet satisfied with their jobs: how do they do it? Child \& Family Social Work, 12, 182-19.

Whittaker, J. K. y Maluccio, A. N. (2002). Issues for program design and outcome Evaluation in Residential Group Child care, en Vecchiato, T.; Maluccio, A. N. y Canali, C. (eds.) Evaluation in Child and Family Services. Comparative Client and Program Perspectives. New Cork: Aldine de Gruyter, 100-119. 


\section{PALABRAS CLAVE}

Teoría de la Autodeterminación, educadores, bienestar, intervención.

\section{KEY WORDS} vention

Self-determination theory, educators, psychological well-being, inter-

\section{PERFIL ACADÉMICO DE LAS AUTORAS}

Marisa García-Merita. Licenciada y Doctora en Psicología por la Universitat de València. Profesora Titular de Personalidad, Evaluación y Tratamientos Psicológicos de la Universitat de Valéncia. Miembro de la Real Academia de Doctores. Su perfil investigador se centra en la Psicología Clínica y de la Salud y en el estudio de la promoción del bienestar.

Isabel Balaguer. Licenciada y Doctora en Psicología por la Universitat de València. Catedrática de Psicología Social de la Universitat de Valéncia. Su principal interés investigador se centra en el estudio de los determinantes psicosociales de los estilos de vida de los adolescentes, en la promoción del bienestar y en los procesos psicosociales en el deporte.

Isabel Castillo. Licenciada y Doctora en Psicología por la Universitat de València. Profesora titular de Psicología Social de la Universitat de València. Su interés de investigación se centra en el estudio de los estilos de vida, el bienestar psicológico, la motivación y la salud, especialmente en la adolescencia.

Marisol Álvarez. Licenciada y Doctora en Psicología por la Universitat de València. Profesora asociada de Psicología Social de la Universitat de València. Formadora en programas de bienestar psicológico. Su interés investigador se centra en la promoción del bienestar y en los procesos psicosociales de los adolescentes.

Lidón Mars. Licenciada en Psicología por la Universitat de València. Master en Rehabilitación e Inserción Psicosocial de la Persona con Trastorno Mental Grave (Título propio de la Universitar de València). 
Dirección de las autoras: Marisa García-Merita, Isabel Balaguer, Isabel Castillo, Marisol Álvarez

y Lidón Mars

Facultad de Psicología.

Avda. Blasco Ibañez, 21

(46010) Valencia

E-mail: Luisa.Garcia-Merita@uv.es

Isabel.Balaguer@uv.es

Isabel.Castillo@uv.es

M.Sol.Alvarez@uv.es

Lidon.Mars@uv.es

Fecha de Recepción del artículo: 2. Febrero 2009

Fecha de Revisión del artículo: 25. Noviembre. 2009

Fecha de Aceptación del artículo: 30. Noviembre. 2009 